\title{
Nouvelles méthodes de calcul pratique des écoulements de filtration non permanents à surface libre
}

\author{
New methods for the practical calculation \\ of non-permanent, free surface, infiltration flow
}

\begin{abstract}
L'élutir des écoulentents de fillration en régime variable est ramené it lit résolation de deux problemes: configuration de l'coulement it wh instant donne et passage de rette configuration ì celle prise par l'econlement it l'instant snimant. les méthodes habituelles des analogies électriques refatives anx dcoulements stationnaires sont ulilisées pour résondre le premier problème (équation de laplace). Les modeles flectriques en papier conducteur ou les réseaux theoblentriques fournissent en particulier les dériueses $\partial \varphi / \partial \mathrm{x}$ et $\partial \mathrm{c} / \partial \mathrm{y}$ sur la surface libre, ce qui permet de calculer les vitesses instantances do déplacement de celle-ci.

Le second problème, qui consiste à passer d'un instant danné $t a ̀$ un instant noisin $t+\delta \mathrm{t}$, est résolu pur unc méthode d'approximations sucressines, yui suppose la mitesse noyenne de díplacement de la surjace libre entre $t$ ef $t+\delta t$ ægale a la demi-somme des vitesses instantanés de déplacement aux temps $\mathrm{t}$ et $\mathrm{t}+\delta \mathrm{t}$.

Différents montages analogiques sont derrits an cours de rette étude, dont la partie thénrique est exposie par M. Schnener of la mise at point des calculs analogiques par M. Huard DF ia Manne.
\end{abstract}

The intestigation of anslealy, free-surface fow of ground-ualer is resumed in the solntion of two problems : form of flom at a given instant and passage form this form to that of the flow in the following instant.

The usual electrical analogy methods concerning stationary flow are employed to solve the first problem (Laplace equation). Electric models mate of conductive paper, or sheo-electric netmorlis, give, in purticular, the derivalines $\partial \uparrow / \partial \mathrm{x}$ and $\partial \varphi / \partial \mathrm{y}$ on the free surface, this enabling the instantaneous velorities of movement of the latter to be calculated. The second problem, which ronsists in passing from a given instunt to the instant immedially following it $t+b t$, is soloed by a method of successine approximations in which the mean velocity of movement of the free surface between $t$ and $t+8 t$ is assumed to equal the half-sum of the instantaneons veloeities of monement at instant $\mathrm{t}$ and $\mathrm{t}+\delta \mathrm{t}$.

Farions analogical circuits are described in this article, of which the theorefical section is presented by Mr. Scuncmint and the analogical culculations developed by Mr. Hrara DE IA Marne.

\section{PREMIERE PARTIE}

\author{
PAR M. SCHNEEBELI,
}

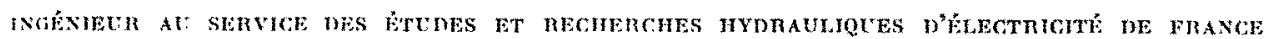

\section{HISTORIQUE}

La théorie de la filtration date de 1856. Sa naissance est marquée par la publication des "Fontaines publiques de la ville de Dijon $*$ de Darct et Ritren.

Ce n'est qu'un demi-siocle plus tard que le problème des écoulements variables ì surface libre fut ahordé pour la première fois. Le fait qu'il lait dé par un des plus éminents savants de l'époque explique que relativement peu de progrès ont été réalisés depuis.
Les travaux de Borssineso sont exposés dons loute une série de notes présentées à l'Acalimic des Sciences entre le 6 juillet 1903 et le 22 août 1904. Ces notes ont été ensuite développées dans deux longs mémoires parus au Journal de Wuthemaliques pures ef appliquees on 1904 .

Boussrnese a montré que dans les eas où les vitesses verticales sont négligeables devant les vilesses horizontales, le probleme pouvait se trai- 
ter analyliquement. En particulier, lorsque l'amplitude des mouvements de la surface libre est faible et négligeable devant l'ápaisseur sensiblement constante de l'écoulement, l'équalion qui régit le mouvement de celte surface est la mèmo que celle qui décrit la propagation non stationnaire de la chaleur dans une barre ou dans une plaque.

Le grand physicien s'est également penehe sur te problème genéral. Il reconnul des 1904 quien lout point se trouvant à l'interieur de l'ecoulement, la fonction $\varphi$, charge hydraulique, salisfait à chaque instant à l'équation de LApLAcE. De même il établit la relation entre les dérivées partielles de $\varphi$ et les dérivées partielles de $h$, cole de la surface libre, qui détermine le monvement de cette surface. Cette équation ne semble malheureusement pas ètre intégrable par les procédés analytiques connus. Boussinesq ne tira de la mise en équation complète du problime que des formules de seconde approximation pour les cas particuliers déjà envisagés ainsi qu'une théorie applicable aux petites dénivellations d'une masse aqueuse infiltrée.

BoussINeso avait pour but essenticl lanalyse du régime des sources alimentées par des nappes phréatiques. Ses successeurs se sont surtout attachés à la transposition de la théorie à d'autres cas d'coulements. Mais aucun des problimes traités ne sont des cas déjà envisagés par le sa- vant français dans lesquels une simplification de l'équation générale permel d'arriver plus ou moins facilement ì une solution analylique. La plupart se réduisent on fin de comple à l'integration de liequation de la chateur.

En 1935, Thuss, aux Elats-Unis, applique celle équation au probleme du pompage dans un puits. Son compatriote Balimax applique, en 1951, la mème théorie aux lerrains d'epandage of aux puits d'injection.

En France, MM. Heaves el Habm reprennent en 1950 les theories de Boussineso ef les appliquent aux nappes de drainage.

Depuis la guerre, il semble qu'en U.R.S.S. une équipe de chercheurs se consacre trìs activement au problème du résime variable. Ainsi PolumaRINova Kotchina introduit le premier blément nouveau depuis Boussineso en tenant compte de l'inertie. Pour les cas déjả envisagés par BousSINESQ, elle arrive à réduire le problème à l'équation télégraphique.

L'examen des coefficients de celle équation montre cependant que le rôle de linerlie est nígligeable, de sorte que le probleme se trouve de nouveau ramené à l'équation de la chaleur. C'est encore cette équation que semblent avoir étudié la plupart des chercheurs russes en vue de son application à différents problemes pratiques. Parmi eux nous citerons Evdokrmova et VERIGIN.

\section{CLASSIFICATION DES PROBLEMES ET OBJET DE CETTE NOTE}

Les écoulements de filtration non permanents à surface libre peuvent se classer on deux catégories principales :

1 Les écoulements pour lesquels les composantes verticales de la vitesse sont négligeables devant les composantes horizontales.

Parmi eux on distingue des problemes it une dimension horizontale et les problèmes à deux dimensions horizonlales. Les premieres peuvent se ramener au probleme de la transmission non stationnaire de la chaleur dian:; une harre, les seconds à la transmission de la chaleur dans une plaque.

$2^{\circ}$ Les écoulements dans lesqueis la composante verticale de la vilesse est sensible.

Ils se subdivisent en éconlements à deax dimensions, dont une verlicale, ef en ecoulements it trois dimensions.

A cette deuxiome categoric de problemes apparliennent presque lous les écoulements créés au voisinage immédial d’ouvrages hydrauliques, tels que tranchées, drains, puits, ete., dès que le rabattement ou le relevement fu niveau d'ean dans ces ouvrages devient important. Il en est de même de l'écoulement crés dans lat rechargo amont d'une digue en tere pro la vidange de la retenue. Tous ces problemes sont reslés jusqu'ici inaccessibles au caleul.

C'est au cours de l'étude di probleme de la vidange d'un barrage en terre que l'ilée nous est venue de passer aux differences finies par rappori à la variable temps ef de ramener ainsi la solution du problème général à une succession de solutions de l'équation de LAplace.

De cette idée nous avons dérive en collahoration etroite avee M. Hand be La Manne, des methodes pratiques de solution de tous les problemes d'écoulement de filtration non permanents à surface libre.

Nous en exposerons ci-dessous les principes thorigues. La mise au point des caleuls analogriques fera l'objel d'un expesé de M. Hrard by ia MarRe. 


\section{COMMENT SE POSE LE PROBLEME}

Dans ce qui va suive, nous traiterons le cas le plus général d'un écoulement à deux dimensions dont l'une verticale. Toules les formules et équations que nous aurons l'occasion d'établir peuvent se transposer sans aucune difficulté aux écoulements à trois dimensions, ear les deux dimensions horizontales interviennent dans la théorie d'une facon symétrique.

Cette limitation a deux dimensions correspond d'une part à un souci de simplification de l'exposé et, d'autre part, au fait que les méthodes de calcul que nous allons décrire n'ont été appliquées jusqu'à présent qu'à des problèmes à deux dimensions. Aucun obstacle theorique ne s'oppose cependant à l'extension de ces méthodes aux ecoulements tridimensionnels et il est permis d'espérer que celte extension se fera dans un avenir proche.

Nous admettrons comme hypothese de base :

- Que l'écoulement suit la loi de Darcy;

- Que le liquide filtrant at le milieu poreux sont ineompressibles;

- Que l'inertie est négligeable. c'est-à-dire que le champ des vitesses réagit immédiatement aux variations de la charge hydranlique.

Cette derniere hypothese est justifice par le fait que les vitesses restent loujours trè faibles ainsi que leurs variations dans le temps.

La première et la troisieme hypothèse se traduisent par les équations:

$$
\begin{aligned}
& u=-K \frac{\partial \varphi}{\partial x} \\
& v=-K \frac{\partial \varphi}{\partial y}
\end{aligned}
$$

Ces équations sont valables à chaque instant, $u$ et $v$ y sont les composantes horizontale el verticale de la vitesse de filtration (débit spécifique), $K$ le coefficient de DARCY et $\hat{\varphi}=p / \circ g+y$ la charge hydraulique.

La deuxième hypothèse se traduit par l'équation de continuité :

$$
\frac{\partial u}{\partial x}+\frac{\partial v}{\partial y}=0
$$

En combinant les équations (1) et (2), on obtient :

$$
\Delta \varphi=0
$$

équation de Laplace valable à chaque instant en tout point de l'écoulement.

Analytiquement, la solution de cette équation est déterminée de façon unique par les conditions régnant à l'instant considéré sur les limites de l'écoulement.

La solution du problème à un instant donné exige donc la connaissance d'une part des limites de l'écoulement, d'autre part des conditions sur les limites.

Ces dernières sont faciles à déterminer par des considérations physiques. On distingue trois types de conditions :

1" La vilesse de filtration est tangente a toute surface impermóable. Sur cetle derniere la condition sera par conséquent :

$$
\frac{\partial \varphi}{\partial n}=0
$$

2' Dans une masse d'eau libre, les pertes de charges sont nulles en comparaison de celles qui règnent à l'intérieur du massif poreux. Une telle masse est done a potentiel constant et la condition a appliquer ă la surface de séparation de l'eau libre el de l'eau contenue dans le massif' est :

$$
=\mathrm{C}^{\mathrm{to}}
$$

$3^{\prime \prime}$ Sur toutes les surfaces le long desquelles l'écoulement de filtration est en contact avec l'air, la pression est égale à la pression atmosphérique $p_{a}=0$. On a done sur ces surfaces la condition :

$$
\varphi=y
$$

Sur la figure 1, la condition (4) est valable le

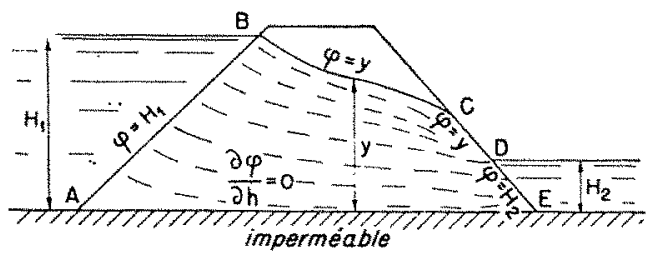

FIG. 1.

long de l'imperméable A E. La condition (5) règne sur A B et D E, surfaces équipotentielles. Enfin la condilion (6) s'applique à la surface libre $B C$ et au segment de suintement $C D$.

On voit que les conditions aux limites sont 
bien définies sur toules les frontières de lécoulement. Pour définir sans ambiguilé la solution du problème à un instant quelconque, il ne reste plus qu'à déterminer le contour, à cel instant, du champ d'écoulement. L'étude d'un écoulement ì surface libre en régime variable se réduit en fin de compte à l'étude du mouvement et de la dé- formation de la surface libre, frontiere mobile de cet ecoulement.

Ce mouvement une fois connu, le probleme se ramene à chaque instant à la solution de l'équation de Laplace pour des conditions aux limites données, tout comme un problème de regime permanent.

\section{MISE EN EQUATION DU MOUVEMENT DE LA SURFACE LIBRE}

Soient deux surfaces libres sucessives (fig. $2 a$ ) correspondant respectivement aux instants $t$ et $t+d t$. Le mouvement de la surface libre d'une position à l'autre se fail sous l'eflet du flux qui la traverse.

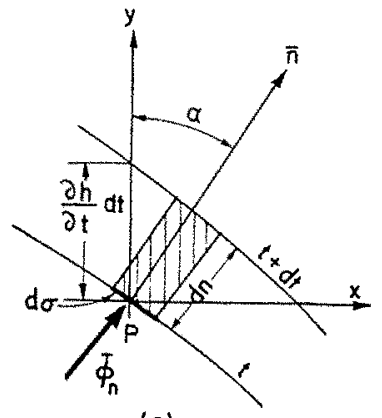

(a)

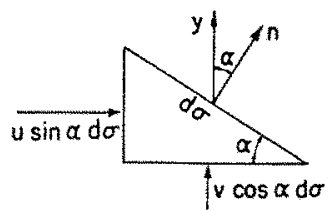

(b)
Fic. 2.

Si nous appelons $\Phi_{n}$ le flux qui traverse l'unile de surface $d$ o de normale $\pi$, nous pouvons ererire que le flux qui la traverse pendant le temps $d t$ est égal au volume des vides du petit parallélépipède $d \sigma d n$ hachuré sur la figure.

$$
\Phi_{n} d \sigma d t=\varepsilon d \sigma d n
$$

où $\varepsilon$ est l'indice des vides du milieu poreux.

L'aceroissement de la cote verticale $h$ de la surface libre at point $P$ de celle derniere est $\partial h / \partial t d t$.

On roit sur la figure que lon a :

$$
d n==\partial h / \partial t d l \cos \%
$$

* dant l'angle aigu que fait la normale $\bar{\pi}$ aree la verticale.

Par ailleurs, le flux qui traverse la pelite surface $d \sigma$ peut s'écrire en appliquant le principe de continuité au petil prisme de la figure $(2 b)$.

$$
W_{n} d a=n \sin x d a+b \cos x d \sigma
$$

où $u$ et $v$ sont les composantes de la ritesse de filtration.
La relation dérivant le monvement de lat surface libre devient donc:

$\varepsilon \partial h / \partial t \cos x d \sigma d t=\| \sin x d \sigma d t+\eta \cos x d \sigma d t$

En $y$ introduisant $\lg z=-\partial h / \partial x$ d les valeurs de $u$ et $v$ donnes par la loi de filtration, on peut mettre celte équation sous la forme:

$$
\frac{\varepsilon}{\mathrm{K}} \frac{\partial h}{\partial t}=\frac{\partial \dot{\varphi}}{\partial x} \cdot \frac{\partial h}{\partial x}-\frac{\partial \rho}{\partial \eta}
$$

Nous obtenons ainsi l'équation aux dérivés partielles qui régit le mouvement de la surface libre. Deux fonctions y figurent. D'une part, l'ble vation $y=h(x, f)$ de la surface libre, dautre part la charge hydraulique $\%(x, y, f)$. Par rap-

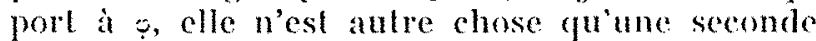
condition a la limite a laquelle doil satisfaire cette fonction. Si le mouvement dail permanent. $\partial h / \partial t=0$, et l'equation (7) deviendrail ha condition de slabilile $\partial_{o} / \partial n=0$, qui, on le sait, dítermine la surface libre en forme ef en position, conjointement avec la condition physique $p=y$.

Nous avons vu prédemment qu'en rúgime non permanent la condition $0=y=h$ itait valable, quel que soit l'instant considere, sur toute la surface libre. Nous pouvons done la dériver soit à $t$ constant le long de cetle surface, soit à $x$ constant en suivant la surface libre dans son mouvement.

La premiere derivation donne:

$$
\frac{\partial x}{\partial x}=\left(1-\frac{\partial y}{\partial !}\right) \frac{\partial h}{\partial x}
$$

of la seconde dérivation :

$$
\frac{\partial \theta}{\partial t}=\left(1-\frac{\partial \omega}{\partial y}\right) \frac{\partial h}{\partial t}
$$

En introduisant ces deux relations dlans l'éfualion $(7)$, on peut en fare disparatitre la fonclion $h$ et icrire:

$$
\frac{\varepsilon}{K} \frac{\partial \rho}{\partial t}=\left(\frac{\partial \theta}{\partial x}\right)^{2}+\left(\frac{\partial \rho}{\partial y}\right)^{2}-\frac{\partial \varphi}{\partial y} \cdot(10)
$$


ef comme s est une fonction harmonique par rapport à $x$ et $y$ :

$$
\frac{\varepsilon}{K} \cdot \frac{\partial \varphi}{\partial t}=\frac{\partial}{\partial x}\left(\cdot \frac{\partial \rho}{\partial x}\right)+\frac{\partial}{\partial y}\left(\frac{\partial}{\partial y}\right) \cdot \frac{\partial \varphi}{\partial y}
$$

Celte dernic̀re forme de l'équation du mouvement de la surface libre est intéressante d'un point de vue théorique car elle se réduit à l'équation de la chaleur pour les problemes de la première catégorie mentionnée au début de cet exposé. En effet, lorsque la composante verticale de la vitesse est négligeable, o devient égal à $h$. L'équation précédente devient :

$$
\frac{\varepsilon}{K} \frac{\partial h}{\partial t}=\frac{\partial}{\partial x}\left(h \frac{\partial h}{\partial x}\right)
$$

el peul se réduire à :

$$
\frac{\varepsilon}{K h} \frac{\partial h}{\partial t}=\frac{\partial 2 h}{\partial x^{2}}
$$

Iorsque les variations de $h$ sont faibles par rapport à $h$.

\section{PRINCIPE DES NOUVELLES METHODES DE CALCUL PRATIQUE}

Considcrons par exemple le massif rectangulaire de la figure 3 . Il est limité de deux còtés par des parois imperméables el en contact le long d'un troisième cóté avec un bassin d'eau libre.

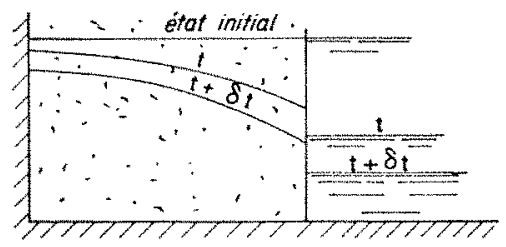

Fic. 3 .

Initialement, le niveau d'eau était au repos dans le bassin. Il clait prolongé à l'inlérieur du massif par une surface libre horizonlale. Lorsque le niveau baisse dans le hassin stivanl une loi donnce, la surface libre descend a linterieur du massif. Supposons connaitre sa posilion a linstant t. Les limiles of les conditions aux limiles de l'écoulement sont ainsi bien déterminées, el la solution de l'équation de Laplacl, obtenue au moyen d'une analogie rhéoélectrique, nous permet d'exaluer, en chaque point de la surface libre, les derivies de la fonetion o au temps $t$. Si nous introduisons dans la formule du déplacement de la surlace libre les valeurs de ces deriveres, elle nous donne les vilesses do rabittement de la surface libe a linstant $l$.

Au bout du lemps of pelit mais fini, la surface libre se trouvera silue en premiere ap- proximation à $(\partial h / \partial I)_{t}$ o $t$ en dessous de sa porsition au temps $t$.

Il est done possible de déduire de la position de la surface libre à un instant donné sa position à l'instant suivant et de détermincr ainsi de proche en proche toutes les surfaces libres successives.

En réalité, on ne se contentera pas de la première approximation, maìs on tiendra également comple de la variation des ritesses de rabaltement d'un instant à linstant suivant. Les methodes de calcu! analogique seront décrites en détail par M. Huard de la Marre qui les a mises au point. Nous signalerons simplement que le raballement lotal pendant le lemps ot peut etre déterminé avec une précision trés salisfaisante puisque l'erreur absolue n'est que de l'ordre de :

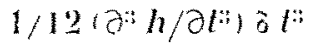

Nous terminerons celle partie de notre exposé en signalamt la généralití absolue de la méthode. Elle peut sappliquer à tous les problemes dont les données sont bien déterminées. Ces problimes peuvent comporter un ou plusieurs plans d'eau libres dont les varialions dans le temps peuvent etre queleonques à condition d'ètre connues. Ils peuvent comporter des zones d'injection ou de soutirage d'un débit constant ou variable dans le temps. Enfin, ancune Jimitation n'est imposce à la forme des massifs conlenant lécoulement of des surfaces imperméables atui le limitent. 


\section{EXTENSION AUX PROBLEMES \\ COMPORTANT DES ZONES DE PERMEABILITES DIFFERENTES ET AUX PROBLEMES D'ÉCOULEMENT EN MILIEU ANISOTROPE}

La présence de zones de perméabilités difrèrenles n'introduit aucune difficulté dans l'application des méthodes décrites ci-dessus.

L'équation :

$$
\frac{\partial}{\partial x}\left(K \frac{\partial \varphi}{\partial x}\right)+\frac{\partial}{\partial y}\left(K \frac{\partial \varphi}{\partial \eta}\right)=0
$$

sera valable en tout point de l'écoulement. Le coefficient $K$ variera cependant d'une zone a l'autre. La solution de cotte équation s'obtient aussi facilement que celle de l'équalion de Laplace au moyen d'un résetu rhélectrique dont les résistanees correspondant aux différentes zones sont inversement proportionnelles aux perméabilités correspondantes.

Il faudra considérer séparément les surfaces libres dans les différentes zones et appliquer i chactune d'elles l'equation $(7)$ avee un coefficient $K / \varepsilon$ différent. On verra alors apparaitre des discontinuités de la surface libre aux passages d'une zone dams lautre, analogue à celles que l'on constate en regime permanent.

Lorsque la permóabilité est anisotrope, elle s'exprime par un lenseur symétrique $\overline{\overline{\mathrm{K}}}$. La loi de filtration écrite par moport aux axes principaux $z_{1}$ et $z_{2}$ devient:

$$
\begin{aligned}
& w_{1}=\cdots K_{1} \frac{\partial \sigma_{0}}{\partial z_{1}} \\
& m_{2}=-K_{2} \frac{\partial \sigma_{2}}{\partial z_{2}}
\end{aligned}
$$

Combiné ave l'équation de continuté, olle donne:

$$
-\frac{\partial}{\partial z_{1}}\left(K_{1} \frac{\partial \theta_{0}}{\partial z_{1}}\right)+\frac{\partial}{\partial z_{2}}\left(K_{2} \cdot \frac{\partial z_{0}}{\partial z_{2}}\right)=0
$$

dont la solution analogique ne présenle pals de difficultes. Il sulfira en effet soil d'utiliser un reseau a mailles carrées orientées selon $z_{1} z_{z}$ et comportant sclon ces directions des résistances proportionnelles a $1 / K_{1}$ et $I / K_{\ldots}$.

On peut également ramener (16) it une équation de Laplace en passant aux variables:

$$
z_{1}^{\prime}=c / \sqrt{\Pi_{1}} z_{1} ; \quad z_{2}^{\prime}=c / \sqrt{K_{2}} z_{2}
$$

L'équation du mouyement de la surface libre érite par rapport aux axes $x, y$, respectivement horizonlal et vertical, est, dans te cas te plus général:

$$
\varepsilon \frac{\partial h}{\partial t}=-u \cdot \frac{\partial h}{\partial x}+v
$$

Ici $u=w_{1} \cos y-\cdots b_{2} \sin \%$

d $b=w_{1} \sin \gamma+w_{2} \cos y$

$\%$ étant l'angle que fait l'axe des $;$ avec l'axe des $x$ complé positivement dans le sens trigonométrique de or vers $0 \approx$.

Nos mathodes de calcul s'appliquent done asallement au cas te plus general d'un beoulement en milicu anisolrope.

Lorsque $x$ coincide aree $z$, of $y$ avee $z$, , ce qui correspond au cas très fréquent di les permearbilités principales sont horizontale el verticale, l'equation du monvement de la surface libre devient :

$$
\varepsilon \frac{\partial h}{\partial t}=\cdots, K_{x} \frac{\partial e}{\partial x} \frac{\partial h}{\partial x} \quad K_{t} \frac{\partial y}{\partial y}
$$

Enfin il va de soi que lorsque l'écoulement prisente des zones de perméabilités différenles dont foutes ou certaines sont anisotropes, les melhom des décrites sonl encore valables.

\section{CONCLUSIONS}

Jusqu'à présent les écoulements à surface libre non permanents n'étaient aceessibles à lanalyse que dans cerlains cas parliculiers. De plus, les developpements mathematiques que demandent les solutions des équations approchées corres- pondant à ces cas peuvent ètre extrèmemont longs pour certaines conditions anx limiles.

Les methodes dont nous arons expose la theoric permettent de resoudre n'importe quel probleme posé. Elles ne nécessitent, en dehers du 
soin apporté à la manipulation du modèle électrique, que des calculs numériques fort simples.

Le progrès qu'elles représentent dans le cadre de la théorie des écoulements de filtration non permanents paraît done pouvoir se comparer à celui qui fut réalisé dans l'étude des problèmes de régime permanent lorsqu'on s'avisa de les ramener à une solution expérimentale de l'équation de LAPLACE.

Bibliographie sommaire

\section{En frangais}

J. Boussineso. - Recherches theoriques sur l'ecoulement des nappes d'eau infiltrés dans le sol et sur le régime des sonres. \& foumal de Mathématiques pures et appliquées $\gg$, tome $\mathrm{X}$, année 1904 , pp. $5-78$ et $363-994$.

L. Hudues et J. Hanm. - Sar les nappes de drainage en régime variable. "Revue générale de l'Hydraulique \$, janvier-férrier 1950 , pp. $24-34$.

\section{En anglais}

C. V. 'THess, - The relation belween the lowering of the piezometric surface and the rate and duration of discharge of a well using ground-mater storage. "Transact, Amerie. Geophysical Union $\geqslant$, aon̂t 1935, pp. 519-524.

P. Bavmaxis. - Ground-water monement controlled through spreading. "Proe. Americ. Soc. Civil Eng. \$, août 1951 , vol. $7 \overline{7}$, separate $n^{\circ} 86$.

En russe

Polvananova Kochisa. - Sur le mouvement non permanent de leau souterraine a partir des réservoirs. "Prikladnaia Matematika i Mekhanika », mars-ayril 1949, pp. 187-206.

Nora. - La documentation en pusse est tres abondante. Elle reste malheureusement peu connue à cause des difficulles de traduction.

\title{
DEUXIEME PARTIE
}

\section{MISE AU POINT DES CALCULS ANALOGIQUES}

\author{
PAR M. P. HUARD DE IA MARRE, \\ ATTACHÉ DE HECHERCHES A L'INSTITUT ULAISE-PASCAL
}

\section{I. - POSITION DU PROBLÈME ET PRINCIPE DE LA MÉTHODE UTILISEE}

Dans l'etude des écoulements de filtration non permanents à surfaces libres, on est amené à résoudre deux problèmes principaux:

$1^{\circ}$ Elant données, à un instant $t_{1}$, la position de la surface libre et celles des différents plans d'eau des bassins déterminant l'écoulement, définir le réseau des équipotenticlles et des lignes de courant, ainsi que les vitesses instantanées de déplacement des différents points de la surface libre.

$2^{\circ}$ Le premier problème étant résolu, passer de la configuration de l'écoulement au temps $t_{1}$ à celle prise par l'écoulement au temps voisin $t_{2}=t_{1}+\delta t$.

On sait que le premier problime est lié à la résolution de l'équation de Laplace $\Delta_{2} p=0$, avec des conditions aux limites qui sont soit de la forme $\rho=y$, soil de la forme $\partial \varphi / \partial_{n}=0$, soit encore $\varphi=\mathrm{C}$. Ce probleme peut done se résoudre aisément par analogie rhéólectripuc, comme un simple écoulement stationnaire.

Les différentes techniques des analogies rhéoélectriques appliquées aux problèmes d'infiltrations ayant été exposées dans une précédente communication, nous n'insisterons pas sur les dćtails de ces techniques $\left({ }^{*}\right)$. Signalons toutefois

(*) «Nouvelles méthodes pour la resolution des écoulements dans les massifs poreux $»$, Mémoires et Trananx de In Sociéte Hydrotechnique de France, $\mathrm{n}^{11} 1,1953$. 
une différence expérimentale entre la représentation de la surface libre d'un écoulement stationnaire et celle d'un écoulement non stationnaire. Dans le premier cas, on ne connail géneralement pas la surface libre, que l'on doit diterminer par approximations successives, mais cette surface est surface de courant.

Au contraire, dans le cas d'un écoulement non stationnaire, cette frontière est supposce connue et l'on doit y imposer une répartition de potentiel $o=y$. La surface libre est alors traversće par un flux.

Rappelons la relation entre les vilesses de déplacement de la surface libre el les dérives de la fonction potentiel o, exposées par M. SchñsBELI. En nous bornant aux écoulements à deux dimensions, cette relation peut se mettre sous l'une des trois formes suivantes:

$$
\begin{aligned}
& \frac{s}{\mathrm{~K}} \cdot \frac{\partial h}{\partial t}=-\frac{\partial \rho}{\partial y}+\frac{\partial h}{\partial x} \cdot \frac{\partial y}{\partial x} \\
& \frac{s}{\mathrm{~K}} \cdot \frac{\partial h}{\partial t}=-\frac{\partial \varphi}{\partial n} \cdot \frac{1}{\cos x} \\
& \frac{\varepsilon}{\mathrm{K}} \cdot \frac{\partial h}{\partial t}=-\frac{\partial \mathrm{w}}{\partial x}
\end{aligned}
$$

avec :

$h=$ cole de la surface libre, $\partial o / \partial n=$ dérivée normale sur la surface libre;

$\alpha=$ angle de la surface libre avee l'horizontale, $\partial h / \partial x=\lg \alpha$

$\Phi=$ flux traversant la surface libre.

Il est facile de constater que ces trois expressions sont équivalentes.

La détermination des vilesses instantances de déplacement de la surface libre en chacun de ses points se ramène donc, selon la relation utilisće, soít à la mesure des dérivées de $\%$ sur cette surface, soit à la mesure de densités de flux la traversant.

Le second problème, qui consiste a passer de la surface libre donnée, relative à linstant $t_{1}$, à la position inconnue de cette surface à l'instant $t_{2}:=t_{1}+\delta t$, met en auvre une méthode d'approximations successives. Soil $\mathrm{M}_{1}$ et $\mathrm{M}_{2}$ les points dintersection des deux positions $S_{1}$ el $S_{.}$

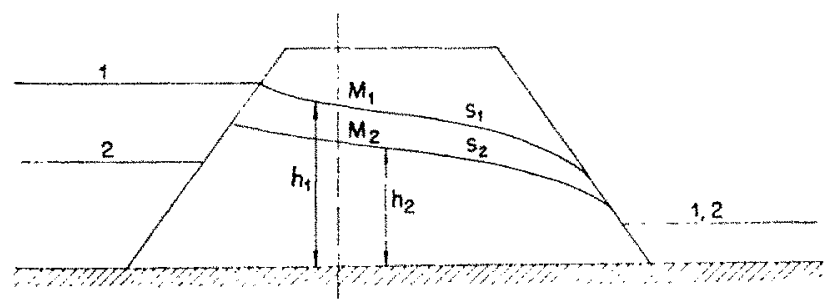

Fig. 1 . de la surface libre aux temps $t_{1}$ d $t_{2}$ avec une méme verticale, $h_{1}$ el $h_{2}$ les cotes de $\mathrm{I}_{1}$ et $\mathrm{M}_{2}$ (voir fig. 1 ).

Nous pouvons écrire:

$$
\begin{aligned}
د h: h_{2}-h_{1} & =\left(\frac{\partial h}{\partial t}\right)_{1} i t+\frac{1}{2}\left(\frac{\partial * h}{\partial t}\right), j l= \\
& +\frac{1}{6}\left(\frac{\partial h}{\partial t^{3}}\right)_{1} \partial t+\ldots
\end{aligned}
$$

D'autre part:

$\left.\frac{\partial " h}{\partial l^{\prime \prime}}\right)_{1} \partial t=\left(\frac{\partial h}{\partial l}\right)_{2}-\left(\frac{\partial h}{\partial t}\right)_{1} \cdots \frac{1}{2}\left(\frac{\partial t h}{\partial t \cdot}\right)_{1} \partial l^{\prime}+\ldots$

En combinant (4) et (5), nous obtenons :

$$
\begin{gathered}
h_{3} \ldots \cdot h_{1}=\frac{1}{2} \Gamma\left(\frac{\partial h}{\partial t}\right)_{1} \\
+\left(\frac{\partial h}{\partial t}\right)_{2} \mid \partial t-\frac{1}{12}\left(\frac{\partial^{\prime} h}{\partial t^{\prime}}\right) \partial t+\ldots
\end{gathered}
$$

En définissant la vitesse moyenne intégrale $t$ :

$$
\left(\frac{\partial h}{\partial t}\right)_{u}=\frac{1}{\partial t} \int_{t_{1}}^{t_{1}+\delta t} \frac{\partial h}{\partial t} d t=\frac{h \cdot \cdots \cdot h_{1}}{\partial t}
$$

l'expression (6) signifie que si l'on prend, dans lintervalle do temps $\left(t_{1}, t_{2}\right)$;

$$
\left.\left.\frac{h_{2} \cdots-h_{1}}{3 t}=\left(\frac{\partial h}{\partial l}\right)_{m}=\frac{1}{2}\right)\left(\frac{\partial h}{\partial l}\right)_{1}+\left(\frac{\partial h}{\partial l}\right)_{2} \mid r\right)
$$

l'erreur ansi faite sur le déplacement verlical $h_{1} \ldots h_{2}$ de la surface libre est de lordre de:

$$
\frac{1}{12}\left(\frac{\partial i h}{\partial t:}\right) \delta t^{:}
$$

que l'on peut considérer comme négligeable si is $t$ est pris suffisamment petit.

Supposons connue la surface libre $S_{1}$, ainsi que la répartition des vilesses ( $\partial h / \partial h)$, le long de $S_{1}$. Il s'agit de déterminer tue surface libre $S_{2}$ telle que son éart rertical, par rapport a $S_{1}$, sa vitesse instantanéc $(\partial / / \partial i)_{2}$, vérificnt en chacun de ses points la relation (7).

Ne connaissant pas $S_{y}$, on se domne foul d'abord une position $S_{2}^{\prime}$ arbitraire (pratiquemenl on peut supposes pour ectle premiere approximation que $\partial h / \partial t$ est constant pendant linlervalle de temps $t_{1}, t_{2}$ et égal à $(\partial h / \partial t)_{1}$. On a alors pour chague point de $s_{1}$ le deplacement :

$$
\Delta h=\left(\frac{\partial h}{\partial l}\right)_{t} \lesssim l
$$

On construit te modele analogique ayant, pour conditions aux limites, la surface libre $S_{2}^{\prime}$ insi 
choisie, ainsi que les hauteurs d'eau des bassins relatives au temps $t_{3}$. Ce modele analogique nous fournit experimentalement la valeur de la vilesse de déplacement vertical $(\partial h / \partial t)_{2}$ en chaque point de $S_{\%}^{\prime}$.

En calculant les valeurs :

$$
\Delta h=\frac{1}{2}\left|\left(\frac{\partial h}{\partial t}\right)_{2}+\left(\frac{\partial h}{\partial t}\right)_{2}\right| \grave{t} t
$$

pour chaque point de $S_{1}$, on détermine a partir de $S_{1}$ une nouvelle surface $S^{\prime \prime}$, , qui serait confondue avec $S_{2}^{\prime}$ si celle dernière occupait la posilion exacte $S_{2}$ de la surface libre au temps $t_{2}$.

Il est facile de voir que $S_{2.2}$ est comprise entre $S_{2}^{\prime}$ et $S^{\prime \prime}$, ; cn effel, si les abaissements $\Delta h$ de la surface libre, ehoisis arbitrairement, et qui déterminent $S^{\prime}$ idans le cas d'un abaissement do nappe ont éte choisis trop faibles, $S_{2}^{\prime}$ se trouve situe au-dessus de la position vraie $S_{2}$, el les vilusses $\partial h / \partial t$ relatives à $S_{:}^{\prime}$ sont supérietures aux vaies valeurs : elles determinent des $\Delta h$ Lrop grands, et la surface $S^{\prime \prime}{ }_{2}$, definie à partir de ces $\triangle h$, se trouve situéce en dessous de $S_{2 .}$

S’il s'agit du cas d'une surface libre remontanle, un raisonnement identique aboulit à la mème conclusion.

Ainsi, avant localise $S$. entre $S_{\text {. }}$ ef $S^{\prime \prime}{ }_{2,}$ on renowvelle le processus en prenant la ligne midiane entre $S_{2}^{\prime}$ et $S^{\prime \prime}$, soil $S^{\prime \prime \prime}{ }_{2}$. On concrétise $\mathrm{S}^{\prime \prime \prime}$ : sur un modele analogicue, d'ou une nouvelle mesure fe la repartition des vitesses $(\partial h / \partial t)_{2}$, el par suite des $₫ h$, ce qui donne, à partir de $\mathrm{S}_{1}$, une nomvelle position $\mathrm{S}^{{ }^{*}}$. Les deux surfaces $\mathrm{S}_{2}^{\prime \prime}$ ef $\mathrm{S}^{\mathrm{T}}$, encadrent encore $\mathrm{S}_{2,}$, et leurs fearls verticaux sont moindres que ceux de $\mathrm{S}_{2}^{\prime}$ et $S^{\prime \prime}{ }_{2}$. On reprend la ligne médiane entre $S_{2} . "$ et $S{ }^{\prime}$, at le procedo est renouvelé jusqu'à ce que les iearts observes soient nigligeables.
Cette méthade converge rapidement. Le nombre dapproximations neessaires, loujours faible, dépend des valeurs relatives du rapport perméabilité sur porosité $k / \varepsilon$ du milieu perméable et de l'intervalle de temps $\delta$ t choisi.

Pour passer d'une surface libre donné $S_{1}$, relalive a un temps $t_{1}$, a la position $S_{n}$ occupec au temps $t_{n}$, il suffira de décomposer lintervalle de temps $\left(t_{1}, t_{n}\right)$ en un nombre suffisamment grand d'intervalles $\delta t$, de facon que l'erreur introduile à chaque étape, soit:

$$
\frac{1}{12}\left(\frac{\partial^{3} h}{\partial t^{3}}\right) \partial t^{3}
$$

ait un maximum suffisamment pelit durant la période $\left(t_{1}, t_{n}\right)$, quel que soil le point considéc de la surface libre.

Le nombre d'étapes permetlant de passer de $S_{1}$ a $S_{n}$ est alors proportionnel à $1 / a t$. Comme l'on commet à chaque dape une erreur de l'ordre de ôt" sur les cotes de la surface libre relativement à la précédente, lerreur définitive sur Jes cotes de $S_{n}$ est au maximum de lordre de $\partial t^{\prime \prime}$, en supposant que ces erreurs s'ajoutent. En réalité ces erreurs ont tendance à se compenser. Prenons par exemple le eas d'une surface lihre qui s'abaisse: si, a the clape, la surface libre trouve est plus haute que la surface réelle, les vitesses mesurées experimentalement le long de cette surface libre sont supérieures aux vitesses correspondant à la surface réelle, et par suile à létape suivante, l'écart a tendance à diminuer. Inversement, si la surface libre trouve est plus basse que la surface relle, les vitesses mesures sont plus fables, et, à l'étape suivante, l'cart entre la surface libre expérimentale et la surface réclle diminue.

\section{II. - CALCULS ANALOGIQUES}

Nous avons utilisé jusquà prèsent deux techniques analogiques, edle du papier conductem et celle du résean de résistances électriques. Celte derniere móthode permet d'étudiar les fooulements en milieux anisotropes of hiterogines. ainsi que les beoulements radiaux, tels que J'ahaissement d'une najpe par pompage dans un puils.

\section{METHODE DI PAPER CONDUCTECR.}

Le modele analogique est decoupé dans une fouille de papier special conducteur, suivant la forme gémitrique du eas cludie (fig. 2).
II est bordé d'électrodes en clinquant de cuivre sur les portions du contour où l'on doit inposer la valeur du potentiel, telles que les por-
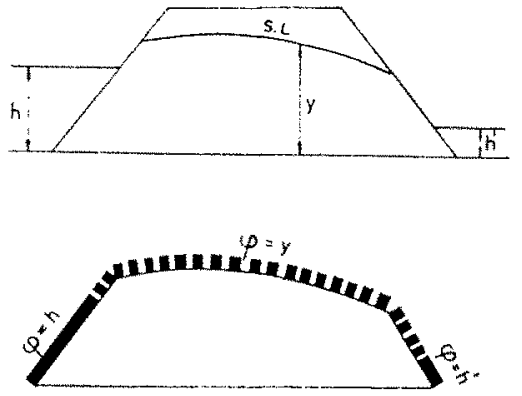

Fix. 2. 
lions en contact avee des zones d'eau libre où le potentiel est égal à la cote du plan d'eau, el les portions de surfaces de suintement el de surfaces libres, où le potentiel est égal à la cote du point considéré. Les portions imperméables du contour sont de simples coupures de papier.

Un tel modèle ayant ses électrodes portées aux polentiels voulus fournit la résolution de l'équation de LAPrace $\Delta_{2} \%=0$ à l'instant considéré, et en particulier les valeurs des dérivées $\partial \rho / \partial x$ el $\partial p / \partial y$ sur la surface libre, ce qui nous donne, à l'aide des relations (1) ol (2), les valeurs de $\partial h / \partial t$. (3) :

Mais il est plus commode d'utiliser la relation

$$
\frac{\partial h}{\partial t}=\frac{K}{\varepsilon} \cdot \frac{\partial y}{\partial x}
$$

où test le flux traversant la surface libre, car la détermination de $\partial \Phi$ revient ì mesurer linlensité du couranl entrant par lélectrode considérée sur la surface libre. Il n'est alors plus besoin de tenir compte de la pente de celle surface.

La méthode du papier conducteur nécessite un modele different pour chaque approximation. Afin d'éviter la rabrication de nombreux modeles analogiques correspondant aux différentes positions de la surface libre, on peut déterminer celle-ci en decoupant plas ou moins profondement le modile analogique suivant des verticales encadrant chaque électrode (fig. 3).

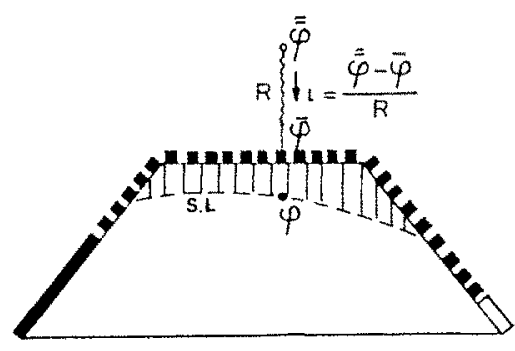

Fia. 3.

On règle le polentiel de chaque électrode de lelle facon que le polentiel sur la surface libre, a la base de la languetle de papier portant cetle blectrode soit égal à $y$. Lees languettes de papier doivent êlre assez ditroites pour que la représentalion de la surface libre soil correcte. Le passage d'une position de celle surface à une autre se fait par simples prolongements des enlailles. Celle méthode n'est évidemment valable que pour l'étude des cas où la surface libre s'abaisse.

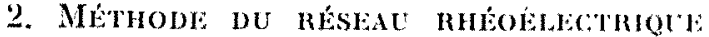 A RÉsistaNCES AMOYHLLS}

Une méthode plus souple consiste at utiliset III réseau rhéoćlectrique à résistances amovibles: la constitution d'un modèle analogique queleonque est alors très rapide, ainsi que le passagge d'une position de la surface libre a une autre.

Sur les bords du modele les mailles ne sont pas entieres, on peul alors donner aux résislanees qui correspondent aux mailles coupites des valeurs proportionnelles aux fractions des mailles (fig. 4). Aux extrémites de ces résistances fractionnaires, le long de la surface libre et des parois de suintement, on impose des valeurs du potentiel correspondant a $\bar{z}=l$.

Rappelons la relation qui existe entre $\partial \varphi / \partial x$, $\partial * / \partial y$ et $\partial h / \partial x$ donl la démonstration est donnée dans l'exposé de M. Schnesmitar :

$$
\left.\frac{\partial y}{\partial x}=1 \quad \frac{\partial \psi}{\partial !}\right) \cdot \frac{\partial h}{\partial x}
$$

En bliminant $\partial_{q} / \partial x$ entre (8) et la relation (1) :

$$
\frac{\varepsilon}{k} \cdot \frac{\partial h}{\partial t} \cdots \cdots \frac{\partial \rho}{\partial !}+\frac{\partial e}{\partial x} \cdot \frac{\partial h}{\partial x}
$$

on obtient :

$$
\frac{\varepsilon}{\mathrm{K}} \cdot \frac{\partial h}{\partial t}=-\frac{\partial \rho}{\partial \eta}+1-\frac{\partial y}{\partial \eta} i
$$

en posant $\partial h / \partial x=i=$ pente de la surface libe.

Ainsi, seule la mesure de $\partial \% / \partial y$ est nécessaire pour déterminer $\partial h / \partial t$ ( $i$ étant connu).

Il est interessant, d'autre part, d'utiliser la variation = definie par :

$$
\tau={ }_{\varepsilon} t
$$

homogine at une longueur, ec qui nous domme l'expression sans dimensions :

$$
\frac{\partial h}{\partial \tau}=-\frac{\partial \varphi}{\partial \eta}+\left(1-\frac{\partial \varphi}{\partial !}\right) i^{2}
$$

En disposant les resistances du réscau horizontalement, les mesures des dérives $\partial \% / \partial \eta$ reviennent à des mesures de differenes de polenliel entre deux nreuds, comme par axemple les nouds 1 et 2 de la ligure 4 . On peut oblenis une meilleure précision en relevant les potentiels de Irois nouds conséculifs, comme par exemple les noxuds 0,1 at 2 de la figure to el en supposint que le potentiel varie, entre ces lrois points, comme un polynôme du deuxième degré. Mais la 
différence observée n'est sensible que pour les régions de forts gradients.

On peut ne pas couper les mailles le long de la surface libre el faire déborder celle-ci par les mailles du réseau : cela revient à prolonger le champ harmonique defini par le modele.

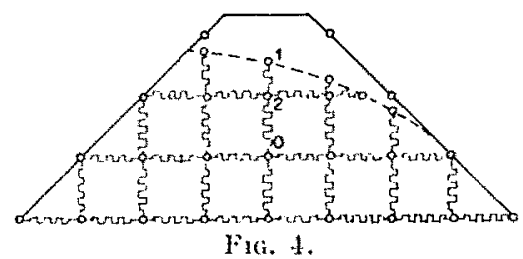

L'imposition des potentiels donnés ôn aux points $M$ donnés de la surface libre se font en agissant sur les potentiels des points 2 extéricurs à la surface libre (fig. 5 et 6 ) : on contròle la valeur du potentiel on $\mathrm{M}$ à l'aide d'un potentiomètre interpolateur branché en parallèle avec la résistance relative à M. Ce potentiomètre
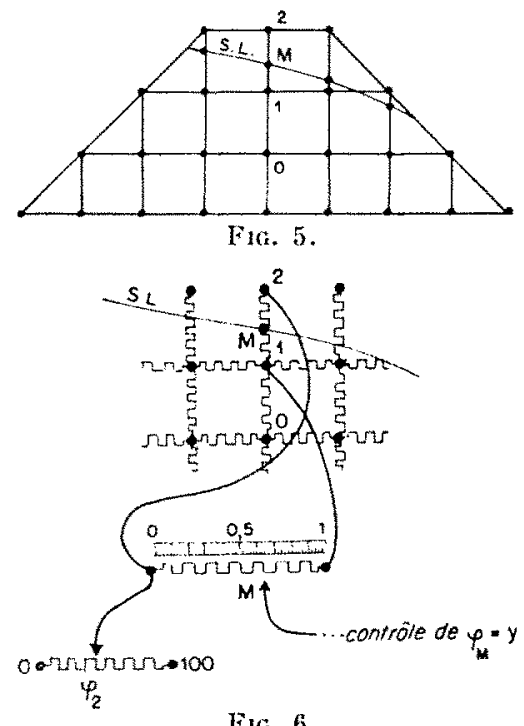

Fig. 6.

doit avoir une impédance très supérieure à celle de la résistance qu'il shunte, afin de ne pas perturber la distribution du potentiel.

Celte methode permet d'uliliser avanlageusement la relation (2):

$$
\frac{\partial h}{\partial \tau}=-\frac{\partial p}{\partial n} \cdot \frac{1}{\cos x}
$$

En efret, on peut exrire, avec les notations de la figure 7 :

$$
\frac{\partial o}{\partial n}=\frac{\theta_{1}-\rho_{I}}{\overline{I J}} \text { et } \cos x=\frac{N^{\prime \prime} J}{I J}
$$

En posant $\mathrm{N}^{\prime \prime} \mathrm{J}=\alpha=$ Iongucur du colé d'une maille du résean, la relation (2) nous donne:

$$
\frac{\partial h}{\partial \tau}=\frac{\vartheta_{1}-p_{I}}{a}
$$

La position de I peul se déterminer soit graphiquement, soil à l'aide de la relation :

$$
\overline{\mathrm{N}^{\prime \prime} \mathrm{I}}-\overline{\mathrm{N}^{\prime} \mathrm{M}^{\prime}}-\overline{\mathrm{N}^{\prime \prime} \mathrm{M}^{\prime \prime}}=a^{\prime}-a^{\prime \prime}
$$

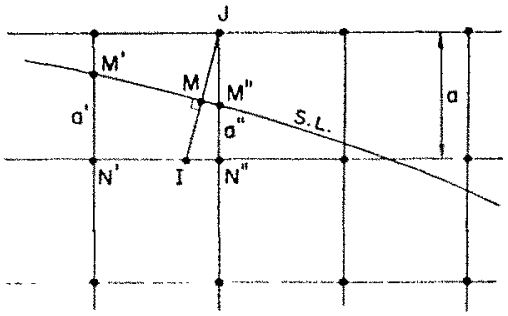

Fia. 7 .

qui donne une précision largement suffisante lorsque la courbure de la surface libre n'est pas trop grande.

Le relevé du potentiel en I se fait à l'aide du potentiomètre interpolateur branché entre $N^{\prime}$ et $N^{\prime \prime}$.

\section{Emplor de math.es rectangelatries.}

Dans certains problèmes, tels que l'élude de l'abaissement d'une nappe de faible épaisseur sous l'effet de pompage par drains, la surface libre présente de faibles variations de cote et, par suite, le potenticl o le long de celle surface varie peu (fig. 8). Dans une telle itude, la hilu-

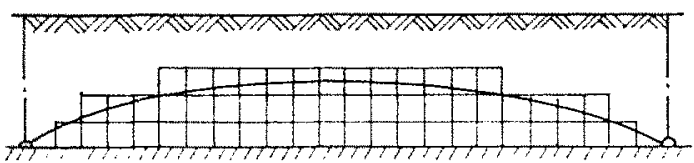

Fia. 8.

teur des mailles doit être petile vu la faible dimension verticale du modèle. L'emploi de mailles carrées aboutirait à un très grand nombre de mailles dans le sens horizontal, d'où un grand nombre de points sur la surface libre nócessitant chacun une imposition de potentiel.

On a alors intérêt à utiliser des mailles rectangulaires plus longues que haules (fig. 9). Les

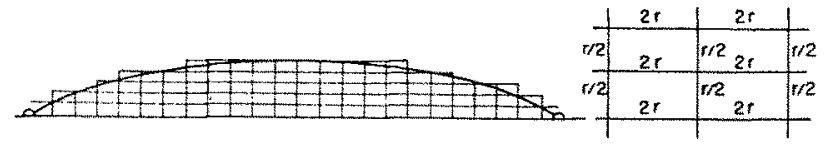

Fig. 9.

résistances horizontales et les résistances rerticales n'ont alors plus la même valeur.

On sait que, étant donnés trois points $A^{\prime}, O, A$ sur un axe $o x$, d'abscisses respectives - $a, 0,+a$, 
et les valeurs $\varphi_{A}$, $\varphi_{0}$, el $\vartheta_{\mathrm{A}}$ d'une fonction $\bullet(x)$ en ces points, on peut écrire, en remplaçant les dérivées par des diflérences finies:

$$
\left(\frac{\partial^{2} \varphi}{\partial x^{2}}\right)_{0}=\frac{1}{a^{2}}\left(\varphi_{\Lambda}+\varphi_{\Lambda}-2 \varphi_{0}\right)
$$

Par suite, si l'on considere cinq points : $0,1,2,3,4$ de coordonnées respectives $(o, o)$, $(a, o),(o, b),(-a, o),(o,-b)$ et les valeurs $\varphi_{0}, \varphi_{1}, \varphi_{2}, \varphi_{3}$ et $\varphi_{4}$ d'une fonction harmonique $o(x, y)$ en ces points, on peut écrire dans les mèmes conditions :

$\Delta_{2} \varphi(x, y)=\frac{1}{a^{2}}\left(\varphi_{1}+\varphi_{3}-2 \varphi_{0}\right)+\frac{1}{b^{2}}\left(\rho_{2}+\varphi_{4}-2 \varphi_{n}\right)$

Celte relation est l'expression de la loi des nœuds dans un réseau électrique à mailles reclangulaires, dont les résistanees horizontales et

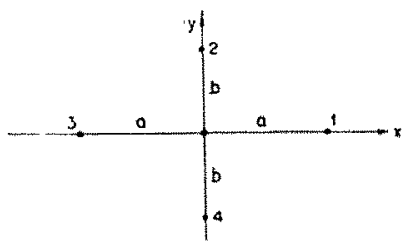

Fio, 10.

verticales ont respectivement pour valeurs $a^{2}$ et $b^{2}$. Le rapport des valeurs des résistances dans un réseau à mailles rectangulaires doil done ètre pris égal au carré du rapport des longueurs (fig. 10).

\section{III. - RESULTATS EXPÉRIMENTAUX}

Nous donnons à titre d'xemple deux cas trailés expérimentalement au réseau rhéoélectrique. Le premier (voir fig. 11) est relatif au drainage l'un massif rectangulaire reposant sur un socle imperméable et limité de part et d'autre par deux tranchées plus ou moins remplies d'eau. Initialement, ces tranchées sont pleines d'eau ef la surface libre dans le massif est horizontale, a la cote 10 . On pompe alors dans les tranches de façon à faire baisser leurs plans d'eau avec

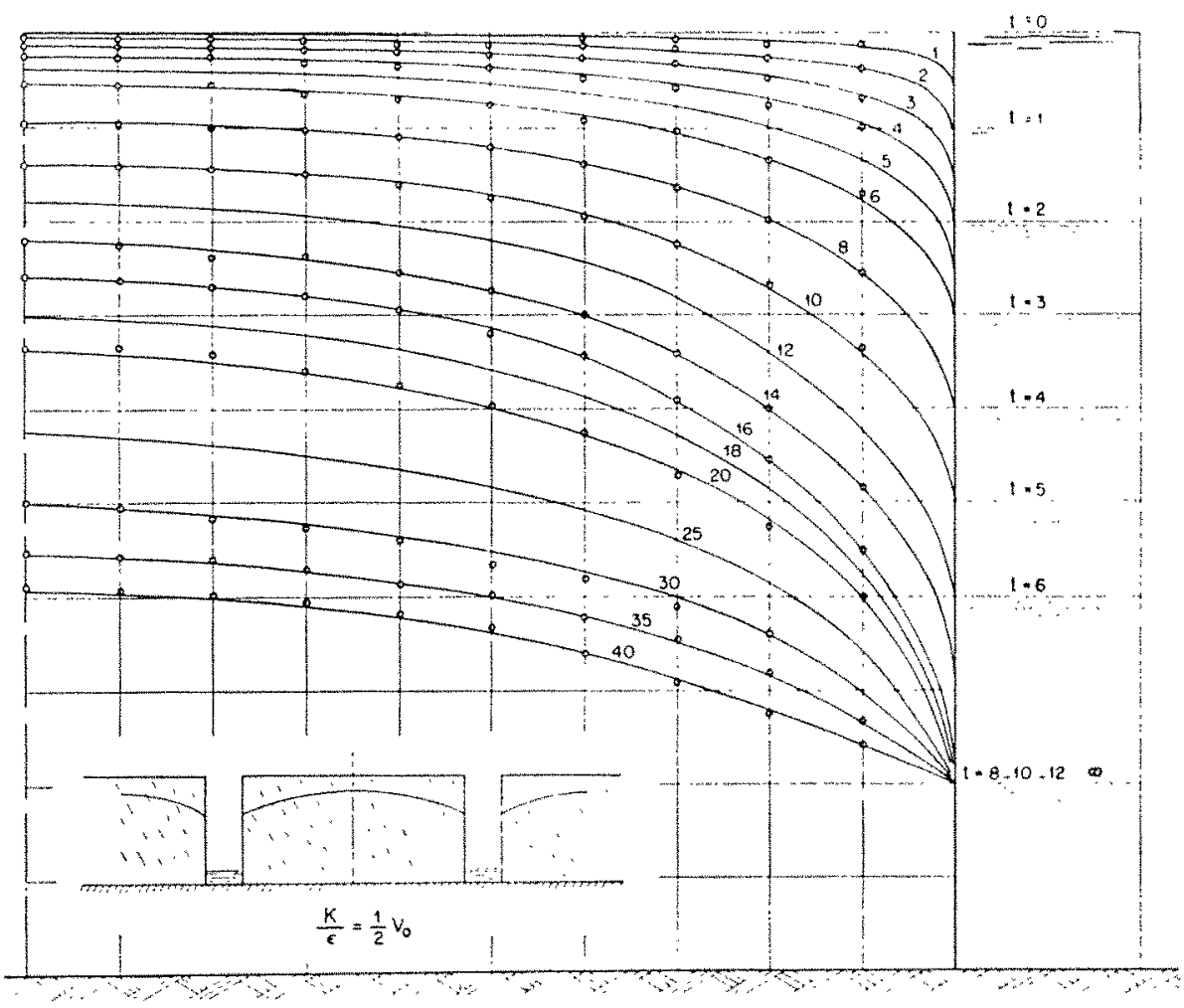

Fia. 11. - Drainage d'un massif rectangulate.

Reaction de la surfuce libre a labaissment du nivea dans les tranchés. 
une vitesse constante $V_{0}$, puis a les slabiliser à la cote 2. Dans cel exemple nous avons pris $V_{0}=2 \mathrm{~K} / \mathrm{s}$.

Les positions des plans deau dans les tranehées et de la surface libre dans le massif, relalives à diférents instants, sont indiquées sur la figure 11, ainsi que les points expérimenlaux ohlenus. Ias seuls fearts nolables sont ceux de la surface relalive au lemps $t=30$, qui a éte ohtenue à parlir de la surface $l=20$. L'inlervalle de temps $\Delta t=\$ 3 \ldots-20:=10$ chail un peu trop fort. Les ecarts sont du reste presque annulés sur la courbe suivante, relative au temps $i=35$.

Le deuxiome exemple (fig. 12) représente le mouvement de la surface libre dans un massil zoné lors de la vidange du bassin amont. Les valeurs de $K / \varepsilon$ dans les différentes zones sont portées sur la figure 12. Dans cet exemple, la valeur de $K / s$ de la recharge amont est tres srande, d'oì la disparition du segment de suintement.

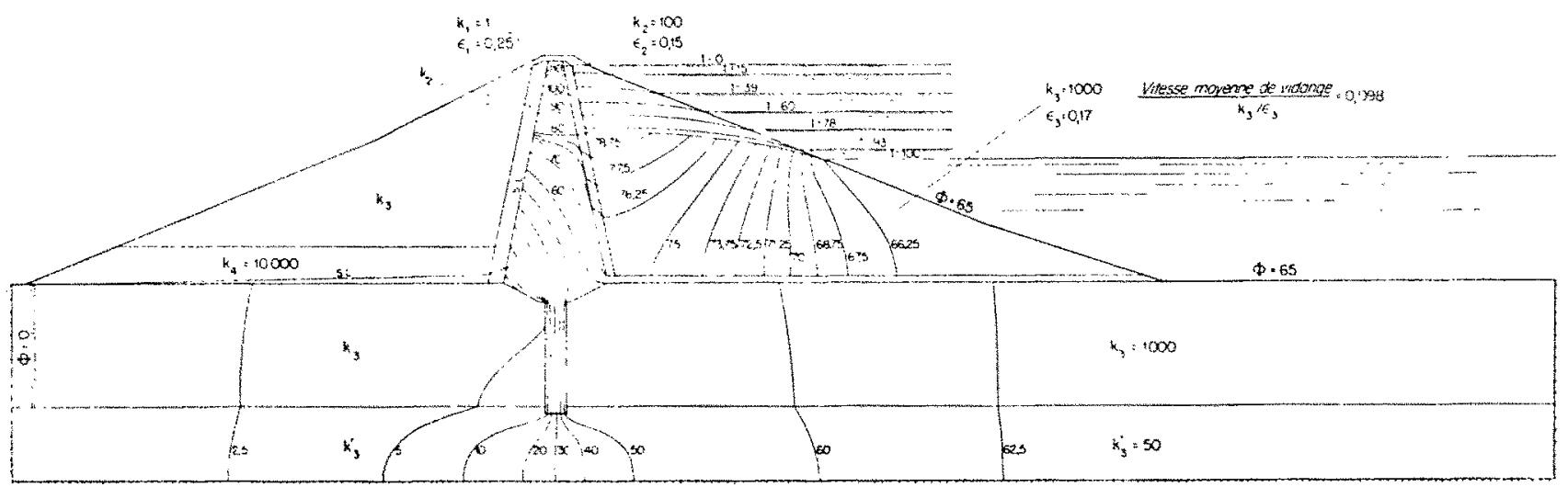

Fic. 12. - Vidange lente de la retenue.

\section{Conclusion}

La méthode que nous venons de proposer pour l'clude des écoulemenls de filtration non stafiomnaires est générale et peut s'appliquer à un srand nombre de problemes de types divers: citons en particulier l'étude de la réaction d'une nappe au pompage d'un puits, les déplacements de la nappe derriere un mur de quai, la stabilité des barrages en terre lor's de la vidange du réservoir, etc, ces problèmes pouvant être relatifs à des milieux anisotropes et hélérogènes.

\section{I S C S S ION}

(Président : M. Gariel)

M. le Président estime que l'exposé de a. Sohnemenel montre, de la part de l'auleur, une parfate connaissanec de l'historique du sujet, une profonde connaissance du sujet lui-meme, et que l'exposé de M. Huarb DE I.A Manne constilue une lllustration tres interessante de lexposé de .M. Schnesbers: il semble qu'à eux deux ils liennent la dé des problèmes des écoulements de filtration.

Tontefois, M. le Président pose deux questions :

1. La methode des approximations suecessives est-elle legitime dans lintegration de ces fonctions qui peuvent presenter des suites convergentes ou divergentes de termes:

2' Dans la baisse de ces surfaces libres, ny a-t-il pas des effets de capillarilé qui, dans le mouvement vertieat, riennent troubler le phenomene hylraulique.

Si ces objections paraissent levées dans une certaine mesure pour les expériences an labomaloire de M. Hoanu Wh LA Marne, est-ce qu'elles ne se presentent pas tontefois dans la réalité des choses?

II. Schxenel répond à la deuxième question que l'effet de la capillarité existe. En première approxmmtion on peut en tenir comple en remplaçant, dans la condition de pression sur la surface libre, la pression atmosphérique (nulle) par la pression capillaire moyenne - Pe développée par les ménisques. Il signale á ce propos qu'il avait étudie en collaboration avee M. Huand Finfluence de celle nouvelle condition aux limites pour un cas de régime permanent. Le résultat qui avait étí obtenu est très intéressant en ce sens que la capillarité n'intervient pas, comme on ladmet parfois, par la criation d'une «frange capillaire $»$ qui se superposerait a l'écoulement, mais modifie d'une façon non négligeable la forme des lignes de courant. Il n'a pas élé fait mention de leffet capillaire dans le cours de lexposé parce que. d'une part, la question de la capillarité est encore ì 
l'élude, et que, d'autre part, la méthode exposce est absolument indépendante de la forme des diférentes condilions aux limites. Il rappelle que, une fois la loi de Darcy admise, l'étude d'un ecoulement de flltation quelconque se mamène á l'etude des conditions aux limites. G'est alors uniquement dans l'expression de ces conditions qu'interviennent les différents facteurs physiques at geometriques.

M. Meyen estime que la tension capillaire joue de plusicurs façons:

1" Par modifation de la pression a la strface libre (changement de pression par tension eapilhare);

2" Par retention d'eau ou d'air.

Ce demier point est, en general, de beancoup le plus important.

En outre, M. Mexen indique un certain nombre d'autres problemes physiques que posent les ecoulements non permanents en milieu poreux.

Si on fait abstraction de ces realites physiques, on pent effectivement réduire le problème des écoulements non permanents a surface libre en milieux poreax à un problème mathématiquement défini. D’ailleurs, il serait facile de donner un grand nombre de solutions analytiques de ce problème en adaptant un certain nombre de résultats connus en fluide parfait. Ainsi, par exemple, le passage d'une houle sous une barrière étudié par Fritz Jown et publie dans Communication on Applied Mathematics, volume 1 , page 149 , peut serrir aux problemes des surpressions derrière les murs de quais. Un article sera d'ailleurs publie prochainement dans $L a$ llouille Blanche à ce sujet.

Il suffit de changer à certains endroits le terme:

$$
\omega^{2} / g \text { en } j \omega / k \quad(j=\sqrt{-1)}
$$

k itant le coefficient de perméabilité.

Il semble qu'aucun chercheur n'ait néanmoins attribué une signifieation physirque à ees ealeuls mathémaliques, puisque personne ne s'est eneore donné la peine de transposer les solutions existantes en fluide parfait.

En ce qui concerne les autres problèmes physiques que posent les ecoulements non permanents et à surface libre en milicux poreux, on peut citer egalement la variation du coeffeient de perméabilité dans le temps pour des terrains fraichement mis en eau (après un certain temps, sourent de lordre do huit à quinze jours, on ateint une valeur asymptotique qui peut etre dans le rapport de une a plusieurs unites avec les raleurs instantanes au commencement); en plus, l'inclusion d'air dans les ecoulements où la surface libre se déplace vers to haut donne lieu a des phénomènes de compressibilité. En plus, il $y$ a parfois des phénomènes physicochimiques importants.

M. Meyer en conclul que l'étude des écoulements non permanents a surface libre dans les milieux poreux est cncore dans le stade de la recherche physique. Il peul être néanmoins très intéressant de savoir à quel résultat mène telle ou telle approximation, mème très grossiere. Si le calcul d'une telle approximation se fait sans peine par transeription simple de formules deja connues, il peut être intéressant. Par contre, si le calcul est long at cher, il ne semble pas justife, étant donné la nature mrossiere des approximations. On est done amene à un probleme économique.

M. Le president remarque que, toutefois, Mal. Sconese-

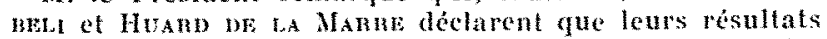
coincident avec la thérie, mas il appert alors des débats qu'it s'agit en réalite d'une coincidence entre les atculs et lear représentation par analogie dedrique, ce qui ne prejuge pas de la coincidence avec la réalite hydraulique.
M. Scanesuen regrelte de ne pouroir présenter des mantenant une justification experimentale de la méthode de calcul proposé. Gelle-ci est en effel très récente et jusqu'à présent il n'a été effectaé qu'un seul essai sur modèle hydraulique. Cel essai devait reproduire un cas analogue a celui du massif reetangulaire dont to calcul analogique a éte présenté par M. Huanb oE wa Manne. Malheurensenent. une fuite dans une paroi étanthe da modele a rendu impossible lat comparaison honnete des résullats. La coineidence des surfaces libres calcule et experimentale semblat ependant aceptable dans la région la plus éloigné de la paroi defectuense.

I. Nexwa propose de comparer les résultats du ealeul analogique on mathematique à des experiences failes avec des malériax de graublometrie suffsamment grosse. Il pense en effet que la encordanee doit atre aussi bome dans le cas de matériaux suffisamment per. ménbles. Les plus grosses divergences doivent avoir lieu dans le cas de sable fin ou d'argile.

M. Huary ne la Mahue mel la question au point en rappelant que la méthode decrite a pour objet de résoudre un probleme mathematique a partir de donnes précises, parmi lesquelles la perméabilité, supposéc constante dans le temps.

\section{Fenrandox exprime sun opinion comme suit:}

* Chacun sait que la Loi de Darey, extension de la Loi de Poisenille a la filtration, constitue une approximation valable entre certaines limites determinant un champ dapplication asse $z$ vaste pour que eclle-ci constitue un moyen propre a faciliter la tache des Ingeniens en prise aux imperieuses necessités de la pratique.

"Le temps at la précision gagnés par les procedes exposés sont déja trés remarquables, puisque les éconlements de l'espece sont regis essentiellement par la perméabilité du terrain contrairement aux coulements permanents qui, dans une certaine mesure on sont independants et que la déterminalion du coeffient de perméabilité est frappée d'une incertitude de base.

Casagnande vient de faire paraitre dans les Transac tions of the American Society of Cibils Engineers (vom lume 117, 1952, page 792) une etude de atermination du temps d'eracuation de leat dans une sous-couche d'acho drone, etablie a partir d'hypotheses bien moins satisfatsantes que celles admises par MM. Scavimust of Hrand, puisqu'il $y$ assimile la surface libre à des droj tes. Neammoins, les resultats de calcul ainsi oblenus, comvenablement verifies par lexperience, se seraient revéles suffisamment exacts pour les besoins de la cause.

M. Antener estime qu'il semail interessant de savoir si les lots et les methodes de calcul exposes restent valahles dans lo eas de mourements mines de la surface libre: mares ou vidanges de réservoirs journaliers. M. Anderer compare le phenoméne a ce qui se passe quand on relite du fravieg d'un lit fluvial an moyen d'une benne; on observe une évacuation tres rapide dune certaine quantite deau qui correspond manifestement it un ecoulement volumique; ensuite lat masse imprégné rend de leau pendant un lemps eneore considerable, mai: suivant une loi tout a fait differente, qui parait correspondre à un ecoulement superficiel. Le rappochement de ces fats dobservation avec la loi des écoulements de filtration semble indiquer que, pendant une periode de transition il y a superposition de deux types d'ecou. lement. Il serait done interessant de roir deja si ce phénomène n'est pas à l'échelle des mares.

Une facon de se rendre comple dans quelle mesure les calculs sont valables dans le cas des mares consisterail a verifer ste phenomene prevu par la lol do Dary est symetrique dans le cas ou leau desend et dans fe cas vit elle monte : si le phinomene de rétention dans ees Chapes differentes de l'ecoulenent lent de l'san it travers les matérianx diffère, c'est que la loi ne s'applique pas. 
M. Schomben remarque qu'en général il s'chablit pendant la descente un segment de suintement entre le plan d'ea et l'inlersection de la surface libre avec la paros filtrante du massif poreux. Ca phenomene ne se retrouve pas dans la montée. Il $y$ a donc dissymetrie des condilions aux limites.

M. Allemer pense qu'il suffirait alors de faire lexpevience pour le cas d'une surface libre se deplagant entre deux parois imperménbles qui diminemient les segmenls de suintement.

A. SoHNebrels confime qu'il existe effeclivement deux modes decoulement ainsi que la fait remarquer M. Autener. En effet, les porosites mesurées par abaissement dans le massif d'un plan d'eau horizontal sont toujours plus fables que celles auxquelles on s'attendail d'après la granulométrie et le mode de mise en place du sable. Ceci s'explique par le fait qu'il reste une certaine quantité deau dans le massif. Cette cau ne s'écoule que très lentement, et il est probable que l'écoulement non saturé ne suit pas la Loi de Darey.

Dans le probleme de la vidange d'un barrage en terre - et c'est an rue de la solution de ce problème que les méthodes de calcul exposées ont été mises au point -... l'eoulement non saturé ne presente eependant qu'un interel atténué puisque dans les zomes qui en sont l. siege, la pression atmosphérique a pu s'établir et que de te fail reau restant retenue sur les surfaces des grains ne peut exereer aucune action nefaste sur la stabilité du talus.

\section{Schneseder répond ensuite à M. Meyer :}

Il fait tout d'abord remarquer qu'il lui paraît un peu difficile de répondre point par point a une intervention aussi volumineuse et portant sur des sujets aussi variés que celle de M. Meyen.

En ce qui concerne l'action de la capillarite. M. Schnerbel estime que sa prise en compte approximative, telle qu'il vient de l'indiquer précédemment, est hien suffisante. L'introduction à la manière de LaMbs (Proceeling A.S.C.E., rolume 76 , septembre 4) de quatre ou cing facteurs pour caractériser la capillarite suivint létal du sol, le mouvement de la surface libre, etc., complique beaucoup le probleme sans qu'il en résulte un avantage pratique en rapport avec cette complication. En effet, dans le cas de la vidange d'un barage en terre, qui nous intéresse particulièrement, les coefficients de perméabilité varient dans le rapport de 1 à 10 suivant les hypothèses. Dans ces conditions d'imprécision de la donné de base qu'est la perméabilité, II semble illusoire de vouloir prendre en compte des donnees secondaires, telles que les variations de la teneur en eau capillaire de la partie vidangée du massif.

M. SCHNenta n'a pas d'objection à l'égard du calcul amalytique tant que les hypotheses simplificatrices, qui sont nécessaires pour que les equations soient integrables, ne sont pas en contradiction avec la nature du problème posé. Il renvoie à ce sujet à la littérature soviétique qui contient un certain nombre d'etudes intéressantes faites sous cet angle. Il estime cependant que la méthode exposée, qui s'applique à n'importe quel problème, garde tout son interet alors qu'une solution analytique est particulière au problème posé. De plus, une solution analytique suppose en général une \&linéarisation $\$ d u$ phénomène qui n'est pas admissible dans le cas général. Il ne paraît pas a priori que les méthodes proposecs par M. MExen échappent à cette règle.

M. Mexen précise que son allusion ne tendait pas opposcr les deux méthodes qui, dans une certaine mesure, se complètent. Il justifie, d'autre part, à M. SchneeBEL certaines transformations d'equations de son interm vention précedente : les problèmes de fluide parfait et les problemes en milieux poreux dont il était question arant no different que par leurs condilions à la surlace libre, et dans ces conditions, le terme :

\section{$\partial^{2} \mathrm{w} / \partial t^{2}$ est simplement remplacé par $\partial \Phi / \partial t$}

Les régimes sinusoidaux donnent done lien a des formules presque identiques; seules quelques constantes réelles deviennent imaginaires et inversement.

En ce quj concerne les régimes non sinusoïdaux, ba méthode elassique qui atilise lintégrale de Fourier donne donc des intégrales immédiatement transcriptibles. Seule l'intégration peut, dans certains cas, être nettement différente.

M. Hepsen demande, si en supposant le coeffieient $k$ connu parfaitement, les methodes proposées donnent des resultats exempts de dispersion, ou presentant une faible dispersion; ou si, au contraire, le mode opératoire ajoute des erreurs a la solution du problème.

M. Huand répond que l'erreur doit être négligeable si on prend des intervalles $\Delta t$ suffisamment petits, c'est-àdire sur un nombre d'étapes suffisamment grand: ce qui serait, toutefois, à vérifier expérimentalement lou inalytiquement).

M. le Président rappelle que cetle réponse s'apparente avec la première des remarques qu'il avait formulee au début de la discussion llégitimation des approximations successives).

H. Schnelisti trappelle que, potr les regimes permanents, l'utilisation du ressau wécélectrique introduit un fractionnement du milieu analytique qui correspond $\dot{a}$ un passage aux différences finies par rapport aux coordonnées de l'espace comparable à celui qui s'effectue par rapport au temps dans les méthodes exposées. Ceci introduit theoriquement des erreurs absolues sur les potentiels de l'ordre de $a^{t}$ ia étant la dimension des mailles). La comparaison de nombreuses solutions de l'équation de Laplace, obtenues au réseau arec celles obtenues à l'aide du papier conducteur, qui n'introduit pas lapproximation mentionnec, montre que ces erreurs sont tout à fait négligeables.

M. Huand ajoute que la précision obtenue dans lexpérience rapportée est de l'ordre de quelques pour cent el qu'il est d'ailleurs possible de prendre un nombre do mailles suffisamment grand pour obtenir un résultat correct.

M. Canes signale que dans un domaine tout à fait différent (pésistance des matériaux), des expériences de rhégraphie faites au Laboratoire des Constructions Navales ont donne une erreur trois a quatre fois plus grande avec le papier conducteur Télédeltos (7 à $10 \%$ qu'avec le bassin à eau ( 2 à $3 \%$ ).

M. Huard rappelle que les calculs faits avec papier conducteur donnaient des écarts de 3 à 4 \% sur les débits dans le cas d'un écoulement permanent.

M. Schneeber ajoute qu'il faut distinguer dans ces écarts la précision électrique des mesures et l'crreur introduite par les approximations successives. Il rappelle que, dans les méthodes décrites, l'crreur introduite par ces dernières dans le passage d'une position de la surface libre à la suivante est de l'ordre de $\Delta \tau^{3}$. Dans le cas du résean $\Delta \tau$ est comparable à la dimension de la maille. L'erreur est done homogène à $a^{3}$. Si le modèle électrique comporte une dizaine de rangés de mailles, cette erreur sera done de l'ordre de $1 / 1.000^{\mathrm{e}}$ de la clarge initiale.

Il faudra a peu près $1 / a$ étapes successives pour obtenir la surface libre finale. En admettant que les erreurs s'ajoutent, ce qui n'est pas forcément le cas, on commettra une erreur globale de $1 / 100^{\circ}$ de la charge inithale sur la position finale de la surface libre. Bien qu'elle soit plus forte que celle qui est introduite par le réseau dans le cas des écoulements permanents (soi1 
$a^{4}$ on $\left.1 / 10.000^{\circ}\right)$, cette creur grlobale parat très acceptable.

M. le Président pense qu'il serait bon de se rendre compte de la précision du procédé in silu: a ce point de vue, les expériences comme celles que II. RËMéviéras va exposer doivent donner des eléments de comparaison.

M. MEYen ajoute que le remplacement des differentielles par des différences finies n'introduit pas de grosse erreur dans le cas ou les frottements sont importants. comme c'est le cas des milieux poreux.

M. Barmllow anrait souhaité et propose pour l'avenir, que la diseussion d'un sujet semblable soit divisée en trois parties : analyse mathematique du phenomène, légitimité du calenl analogique el de ses divers procédés (papier conducteur, réseaux, ete.) à partir de données supposées exactes et aspect physique ou expérimental du phénomène, tel que l'observent les Ingénieurs de la filtration (en particulier la variabilité du coefficient do perméabilité).

En ce qui concerne les considerations physico-chimiques, N. Bammorox renroie au cours d'Hydraulique de M. Trsox, dans lequel on trouve toutes descriptions des divers états de l'eau dans le sol.

M. le Président remarque que la question tratee présentera le plus grand interêt pour les «Joumés de FHydraulique $\gg$ de 195t, qui seront consacrées aux études d'ecoulement souterrain et auxquelles seront invites des savants étrangers: il demande done aux participants à la discussion de ee jour d'échanger entre temps leurs idées sur le sujet discuté en vue d'aboutir a la présentation aux *Journées de l'Hydraulique d'un ensemble cohérent des points de vue des protagonistes de la Société Hydrotechnique de France.

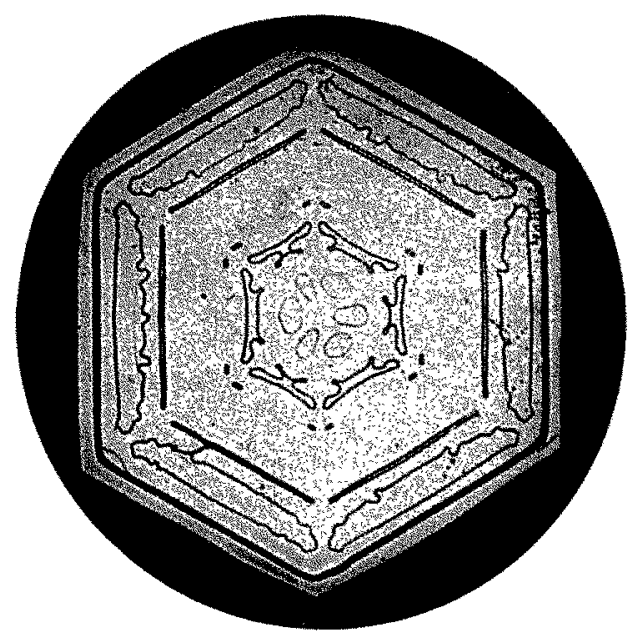

